\title{
Is the human race winning the war against Helicobacter infections?
}

\author{
Joseph 0. Ashaolu* \\ International Health Programme, School of Medicine, National Yang-Ming University, Taipei, Taiwan. \\ E-mail: noblebidemee@gmail.com
}

\begin{abstract}
The incidence of helicobacter infections in human population appears not to have significantly wane over the years. Even though there are reports indicating that the prevalence rate is reducing in the developed world, constant migration, general globalization and other environmental factors has kept every population a risk. Moreover, the rising incidence of infection with non-pylori helicobacter species, which were considered hitherto non-virulent, puts a question in the seemingly progress made so far in the treatment and eradication of these bacteria and their infections. The purpose of this mini review is to access the clinical and epidemiological gains and the challenges so far in the treatment and eradication of helicobacter spp. infections.
\end{abstract}

Keywords: helicobacter spp.; infections; human population; bacteria; eradication

\section{INTRODUCTION}

Helicobacteria, a group in the Epsilonproteobacteria, colonize and infect the gastrointestinal tracts of a large variety of animal hosts [1]. Presently, there are about 35 species in the helicobacter genus. Helicobacter pylori, which is the most significant Helicobacter species has been implicated as an important aetiological factor in a variety of human gastric disorders, such as duodenal ulcer, gastric ulcers and gastric cancer [2].

Recent data shows that the prevalence of $H$. pylori infection in humans is more than half the world's population even though with large amounts of variation among regions. For instance,

it was reported that the regions with the highest $H$. Pylori prevalence were Africa (70.1\%), South America (69.4\%), and Western Asia (66.6\%) while regions with the lowest reported HP prevalence were the Oceania (24.4\%), Western Europe (34.3\%), and Northern America (37.1\%), respectively [3]. Although not all these over 4.4 billion infected people are symptomatic, however, they are all potential cases of individuals who might become clinical cases in the long run.

It is noteworthy that at the turn of the 21st century, the prevalence of $H$. pylori infection has been declining in highly industrialized countries of the Western world, whereas prevalence perpetually remains at a high level in developing and newly industrialized countries. The widening differential gap in prevalence has important implications on the future worldwide prevalence of sequelae associated with $H$. pylori, including peptic ulcer disease and gastric cancer [3].

Furthermore, even though the helicobacter infections are often acquired at childhood, recent meta-analysis reveals that the prevalence of the infection in adults ( $\geq 18$ years) was significantly higher than in children
(48.6\% vs $32.6 \%$, respectively). A trend analysis also indicated that there was a statistically non-significant decrease in the prevalence of $H$. pylori infection from 2009-2016 compared with the 2000-2009 period.

From the foregoing therefore, it is pertinent to examine if actual progress is being made in the eradication of this bacteria and its associated outcome in the human population.

\section{'Traditional' Diseases Associated with Helicobacter Pylori Gastric and Duodenal ulcers \\ Till date, the mechanism by which $H$. pylori promotes duodenal ulceration is not completely understood. However, the leading hypothesis is that H. pylori induces high levels of gastric acid secretion in the antrum. This leads to the replacement of intestinal-type tissue in the duodenum with gastric tissue. The overall effect culminates in the allowance of $H$. pylori in colonizing the areas of gastric metaplasia and together with elevated acid levels, ultimately promote ulceration in this locale [4].}

Clinical data has shown that $95 \%$ of duodenal and $70 \%$ of gastric ulcers are associated with Helicobacter pylori. Incidentally, Peptic ulcer disease is the cause of dyspepsia in about $10 \%$ of people [5]. These authors also submit that $H$. pylori eradication therapy is effective in preventing recurrence of duodenal and gastric ulcer (ulcer returning after initial healing) compared to no treatment. There is currently no evidence that $H$. pylori eradication therapy is an effective treatment in people with gastric ulcer or that it is effective in preventing recurrence of duodenal ulcer compared to ulcer-healing drugs [5]. There is a mix in the multifactorial causes, and therefore, the need for a long-term cohort studies and/or studies with enough statistical power to ascertain the role of helicobacter eradication in the aetiology of gastric ulcer reoccurrence. 


\section{Gastric cancer}

Helicobacter pylori has been implicated in the initiation and progression of gastric cancer through several mechanisms. For instance, chronic gastric inflammation, progressing to the precancerous changes of atrophic gastritis and intestinal metaplasia has been an identified pathway. The extent and/or severity of these precancerous changes has a direct relationship with the risk of developing gastric cancer [6]. Another mechanism reported through which Chronic H.pylori contributes to gastric mucosal genetic instability is by reducing gastric acid secretion (hypochlorhydria). This reduction has a potential of promoting the growth of gastric microbiome that processes dietary components into carcinogens [7].

Gastric cancer is a major global health threat and is the third leading cause of cancer deaths worldwide causing an estimated $>720,000$ deaths per year globally [8]. Even though it has been suggested that the eradication of $H$. pylori, using some antibiotics regimen, can result in resolution of gastric inflammation, halt the progression of gastric mucosal damage, prevent further $H$. pylori-induced DNA damage, improve gastric acid secretion, and restore the microbiome toward normal [7], the result has been conflicting with uncertainty in its generalization across the globe, especially across the high risk groups including the low-incidence populations [9]. Moreover, the best of such antibiotic therapeutic application, from global clinical data, has resulted in only a significant reduction (54\%) in the incidence of gastric cancer. It has been speculated that without effective preventive measures, the current high incidence of gastric cancer will remain stable or even increase by 2030 [8]. Consequently, the International Agency for Research on Cancer (IARC) has indicated the urgent need for effective preventive measures and for a critical assessment of $H$. pylori eradication as a preventive strategy [10].

\section{The advent of infections from non-H. pylori helicobacter spp.}

Recently, the number of clinical cases reported due to non-pylori helicobacter spp. gives some level of concerns. For instance, in a PCR-based investigation, it was found that the presence of $H$. pylori DNA was associated with acute gallstone cholecystitis in some patients [11]. In addition, a clinical report indicated that Helicobacter bilis was overrepresented in poorly differentiated cancers as compared to welldifferentiated cancers which also implies that $H$. bilis is potentially associated with a poorer differentiation and more negative prognosis in patients with colorectal cancer [12]. In a child from Cambodia, Helicobacter fennelliae was isolated from a nondiarrheal stool sample. However, the infecting strain showed resistance to macrolides and quinolones, the first-line antibiotic therapy for Campylobacter-like infections [13].

\section{The challenges with eradication}

The present challenges with helicobacter infections are numerous. These challenges include but not limited to antibiotic resistance [14], reoccurrence of infection [15], behavioural and lifestyle of individuals such as observance of standard hygiene and sanitary practices [15], including careful interaction with pet (for pet owners) and other animals in order to avoid zoonotic transmission of the bacteria[16]. All these factors have to be properly situated in order to ensure adequate protection in the human population.
In a study for example, the global annual recurrence, reinfection and recrudescence rate of $\mathrm{H}$. pylori were found to be $4.3 \%, 3.1 \%$ and $2.2 \%$, respectively. Interestingly, these recurrence rates remained relatively stable between 1990s, 2000s and 2010s although varied across different regions [15].

\section{CONCLUSION}

It does appear there is still a long way to go in the eradication of helicobacter infections. Special attention however needs to be given to developing and underdeveloped nations as they may serve as the rate-determining step in the eradication of these longterm human microbiota occupier.

\section{ACKNOWLEDGMENTS}

The work was self-supported by the author.

\section{REFERENCES}

[1] Euzéby, J. P. (1997). List of Bacterial Names with Standing in Nomenclature: a folder available on the Internet. International Journal of Systematic and Evolutionary Microbiology, 47(2), 590-592.

[2] Sanders, M. K., \& Peura, D. A. (2002). Helicobacter pylori-associated diseases. Current gastroenterology reports, 4(6), 448-454.

[3] Hooi, J. K., Lai, W. Y., Ng, W. K., Suen, M. M., Underwood, F. E., Tanyingoh, D., ... \& Chan, F. K. (2017). Global prevalence of Helicobacter pylori infection: systematic review and metaanalysis. Gastroenterology, 153(2), 420-429. doi: 10.1053/j.gastro.2017.04.022

[4] Varga, M. G., \& Epplein, M. (2019). Helicobacter Pylori-Mediated Carcinogenesis. 87-197. doi.org/10.1016/B978-0-12-801238-3.65172-3

[5] Ford, A. C., Delaney, B., Forman, D., \& Moayyedi, P. (2006). Eradication therapy for peptic ulcer disease in Helicobacter pylori positive patients. Cochrane Database of Systematic Reviews, 4(4):CD003840. doi: 10.1002/14651858.CD003840.pub5.

[6] Correa, P. (2004). The biological model of gastric carcinogenesis. IARC scientific publications, (157), 301-310.

[7] Machado, A. M. D., Figueiredo, C., Touati, E., Maximo, V., Sousa, S., Michel, V., ... \& Rasmussen, L. J. (2009). Helicobacter pylori infection induces genetic instability of nuclear and mitochondrial DNA in gastric cells. Clinical Cancer Research, 15(9), 2995-3002.

[8] IARC Helicobacter pylori Working Group. Helicobacter pylori Eradication as a Strategy for Gastric Cancer Prevention. Lyon, France: International Agency for Research on Cancer (IARC Working Group Reports, No. 8). Available at: http://www.iarc.fr/en/publications/pdfs-online/ wrk/wrk8/index.php. Accessed on November 21, 2015

[9] Lee, Y. C., Chiang, T. H., Chou, C. K., Tu, Y. K., Liao, W. C., Wu, M. S., \& Graham, D. Y. (2016). Association between Helicobacter pylori eradication and gastric cancer incidence: a systematic review and metaanalysis. Gastroenterology, 150(5), 1113-1124. doi: 10.1053/j.gastro.2016.01.028

[10] Herrero, R., Parsonnet, J., \& Greenberg, E. R. (2014). Prevention of gastric cancer. JAMA, 312:1197-1198.

[11] Fatemi, S. M., Doosti, A., Shokri, D., Ghorbani-Dalini, S., Molazadeh, M., Tavakoli, H., ... \& Tavakkoli, H. (2018). Is there a correlation between Helicobacter pylori and enterohepatic Helicobacter species and 
gallstone cholecystitis?.Middle East journal of digestive diseases, 10(1), 24.

[12] Wu, Y., Shi, L., Li, Q., Wu, J., Peng, W., Li, H., ... \& Fu, X. (2019). Microbiota diversity in human colorectal cancer tissues is associated with clinicopathological features. Nutrition and cancer, 71(2), 214-222.

[13] Ruksasiri, S., Lurchachaiwong, W., Wassanarungroj, P., Serichantalergs, O., Sivhour, C., Samon, N., ... \& Crawford, J. (2018). Antimicrobial resistant Helicobacter fennelliae isolated from non-diarrheal child stool sample in Battambang, Cambodia. Gut pathogens, 10(1), 1-5.

[14] Drossman, D. A., \& Hasler, W. L. (2016). Rome IVfunctional GI disorders: disorders of gut-brain interaction. Gastroenterology, 150(6), 1257-1261.

[15] Hu, Y., Wan, J. H., Li, X. Y., Zhu, Y., Graham, D. Y., \& Lu, N. H. (2017). Systematic review with meta-analysis: the global recurrence rate of Helicobacter pylori. Alimentary pharmacology \& therapeutics, 46(9), 773-779. doi:10.1111/apt.14319

[16] Mladenova-Hristova, I., Grekova, O., \& Patel, A. (2017). Zoonotic potential of Helicobacter spp.Journal of Microbiology, Immunology and Infection, 50(3), 265-269. 